\title{
3C 129: The GMRT observations
}

\author{
Dharam Vir Lal ${ }^{1}$ and A. Pramesh Rao ${ }^{1}$ \\ National Centre for Radio Astrophysics, Pune University campus, Ganeshkhind, Pune 411 007, India \\ e-mail: dharam@ncra.tifr.res.in; pramesh@ncra.tifr.res.in
}

Received 1 December 2003 / Accepted 8 March 2004

\begin{abstract}
We present radio maps of the head-tail radio galaxy, 3C 129 at 240 and $610 \mathrm{MHz}$ using the Giant Metrewave Radio Telescope. We have studied in detail the morphology of the source and the distribution of spectral index over the object. We find large-scale spectral steepening along the jet away from the core. Using synchrotron spectral ageing theory, we infer the age (time since particle acceleration) of electrons at various locations along the jet and ascribe an age of $\sim 200 \mathrm{Myr}$ to 3C 129. We find that the "Crosspiece" has a spectral index of $\sim-1$, similar to the jet, which is not consistent with the suggestion by Lane et al. (2002) that it could be a radio relic. We have also studied 3C 129.1 and the other sources in the field.
\end{abstract}

Key words. galaxies: active - galaxies: clusters: individual: 3C 129 - radio continuum: galaxies

\section{Introduction}

Head-tail radio sources, which are almost always associated with clusters of galaxies, are characterised by a highly elongated radio structure with the associated optical (usually giant elliptical) galaxy at one end. A classical example of this class is 3C 129 which, along with its companion 3C 129.1 (a wide-angle-tail), is a member of the $\mathrm{X}$-ray cluster $4 \mathrm{U} 0446+44(z=0.021)$ that happens to lie in the galactic plane $\left(b=0.5, l=160^{\circ}\right.$ ) (Macdonald et al. 1966; Hill \& Longair 1971). 3C 129 is classified as a prototype narrow-angle-tail (Lane et al. 2002) and has a total flux density of $\sim 6.27 \mathrm{Jy}$ at $1400 \mathrm{MHz}$ (Bologna et al. 1969). This very long narrow tailed source has been studied at various wavelengths and with different resolutions (Owen et al. 1979; Downes 1984; Perley \& Erickson 1979; Rudnick \& Burns 1981; Jägers \& de Grijp 1983). Recently this source was studied by Lane et al. (2002) at $330 \mathrm{MHz}$ which is the lowest radio frequency at which it has been observed.

A variety of models have been proposed to explain the morphology and spectral index distribution of the head-tail sources and 3C 129 in particular (Jaffe \& Perola 1973; Pacholczyk \& Scott 1976; Krawczynski et al. 2003). In spite of possible systematic errors due to differing UV coverages and possible artifacts introduced by deconvolution procedures, meaningful conclusions have been drawn about the morphology of 3C 129 and its spectral index distribution. The main result is that the radio spectrum has been found to steepen with distance $\left(S_{v} \propto v^{\alpha}\right.$, where $S_{v}$ and $\alpha$ are the flux density at frequency $v$ and the spectral index

Send offprint requests to: Dharam Vir Lal, e-mail: dharam@ncra.tifr.res.in respectively) along the tail of 3C 129 (Jaffe \& Perola 1973; Miley 1980; van Breugel \& Willis 1981) and has been interpreted in terms of ageing of electron population. A variant of the Pacholczyk \& Scott (1976) model was suggested by Perley \& Erickson (1979), who suggested that the longer wavelength spectrum (between 74 and $1415 \mathrm{MHz}$ ) in any particular location of the tail $\gtrsim 6^{\prime}$ from the head, is straight (a single power law) but steadily steepens with increasing distance from the head. Both these observations can well be explained using a combination of continuous injection of new particles (thus resupplying the high-energy electrons) and particle diffusion out of the source (thus preventing accumulation of low-energy particles). This led to the refined KardashevPacholczyk (Kardashev 1962; Pacholczyk 1970) and JaffePerola (Jaffe \& Perola 1973) models.

Recent high resolution observations by Lane et al. (2002) at $330 \mathrm{MHz}$ have shown a small perpendicular object, referred to as the "Crosspiece", near the head of the galaxy. This feature has a steep spectrum $\left(\alpha_{330}^{610}=-3 ;-1.5<\alpha_{330}^{1400}<-1.1\right)$ and is interpreted by them as a pre-existing fossil radio source that was revived due to $3 \mathrm{C} 129$ ploughing through it. The relic source is compressed by the bow shock of 3C 129 causing it to radiate behind the shock front, producing the characteristic shape. If this interpretation is correct, structures like the crosspiece should be rare which is verifiable by making high resolution low frequency maps of a well defined sample of head-tail sources.

We have started a project of studying a sample of head-tail sources at 240 and $610 \mathrm{MHz}$ using the GMRT. In this paper we present results for 3C 129 and describe the morphological and spectral properties. We use the data to study the behaviour of the low frequency spectra along the tail and compare it with the 
existing models. We confirm the existence of some of the steep spectrum background sources reported by Lane et al. (2002) at $330 \mathrm{MHz}$.

\section{Observations}

We made full synthesis observations of 3C 129 at 240 and $610 \mathrm{MHz}$ using the GMRT on 19 and 24 May 2002 respectively in the standard spectral line mode with a spectral resolution of $128 \mathrm{kHz}$. Table 1 gives the details of the observations. The visibility data were converted to FITS and analysed using standard AIPS. The flux calibrator 3C 147 was also the amplitude and phase calibrator and was observed once every 40 min. 3C 147 was used to estimate and correct for the bandpass shape and to set the flux density scale which is an extension of the Baars et al. (1977) scale to low frequencies, using the coefficients in the AIPS task "SETJY". The error in the estimated flux density, both due to calibration and systematic, is $\$ 5 \%$. In addition to normal editing of the data, the observations at $240 \mathrm{MHz}$ were affected by intermittent radio frequency interference (RFI) and channels affected due to RFI were identified and edited, after which the central channels were averaged using "SPLAT" to reduce the data volume. To avoid bandwidth smearing, $5 \mathrm{MHz}$ of clean band at $240 \mathrm{MHz}$ was reduced to 4 channels of $1.25 \mathrm{MHz}$ each. At $610 \mathrm{MHz}$ where there was little RFI, $12.75 \mathrm{MHz}$ of clean band was averaged to give 2 channels of $6.375 \mathrm{MHz}$ each.

While imaging, 37 facets (obtained using AIPS task "SETFC"), spread across $\sim 2^{\circ} \times 2^{\circ}$ field were used at $240 \mathrm{MHz}$ and 5 facets covering slightly less than $0.7 \times 0.7$ field, were used at $610 \mathrm{MHz}$ to map the field using the AIPS task "IMAGR". We used "uniform" weighting and the 3-D option for $\mathrm{W}$ term correction throughout our analysis. The presence of a large number of point sources in the field allowed us to do phase self-calibration to improve the image. After 2-3 rounds of phase self-calibration, a final self-calibration of both amplitude and phase was made to get the final image. At each round of self-calibration, the image and the visibilities were compared to check for the improvement in the source model. The final maps were combined using "FLATN" and corrected for the primary beam of the GMRT antennas.

\section{Radio maps}

The high angular resolution map of 3C 129 at the lowest frequency of $240 \mathrm{MHz}$ is shown in Fig. 1. Figure 2 shows the image at $610 \mathrm{MHz}$. The images shown have nearly complete UV coverage, an angular resolution $12^{\prime \prime}$ and $7^{\prime \prime}$ and the rms noise in the maps are $\sim 1.3 \mathrm{mJy}^{\text {beam }}{ }^{-1}$ and $\sim 0.3 \mathrm{mJy}_{\text {beam }}{ }^{-1}$ at 240 and $610 \mathrm{MHz}$ respectively. The dynamic range in each of the maps is $\gtrsim 400$ (see also Table 1). The GMRT has a hybrid configuration (Swarup et al. 1991; Ananthakrishnan \& Rao 2002) with 14 of its 30 antennas located in a central compact array with size $\sim 1.1 \mathrm{~km}$ and the remaining antennas distributed in a roughly "Y" shaped configuration, giving a maximum baseline length of $\sim 25 \mathrm{~km}$. The hybrid configuration gives reasonably good sensitivity for both compact and extended sources. The baselines obtained from antennas in the central square are
Table 1. Observing $\log$ for $3 \mathrm{C} 129$. Centre of the field was at $\mathrm{RA}_{\mathrm{B} 1950}=044525.06$ and $\operatorname{Dec}_{\mathrm{B} 1950}=445700.09$ and use 86.5 Mpc $\left(H_{0}=71 \mathrm{~km} \mathrm{~s}^{-1} \mathrm{Mpc}^{-1}\right)$ as the distance to 3C 129 .

\begin{tabular}{l|cc}
\hline \hline & $610 \mathrm{MHz}$ & $240 \mathrm{MHz}$ \\
& & \\
\hline & & \\
Observing date & $24 \mathrm{May} 2002$ & $19 \mathrm{May} 2002$ \\
Duration & $7.45 \mathrm{~h}$ & $6.84 \mathrm{~h}$ \\
Centre frequency & $613.375 \mathrm{MHz}$ & $242.062 \mathrm{MHz}$ \\
Nominal bandwidth & $16 \mathrm{MHz}$ & $8 \mathrm{MHz}$ \\
Effective bandwidth & $12.75 \mathrm{MHz}$ & $5 \mathrm{MHz}$ \\
Primary beam & $43^{\prime}$ & $108^{\prime}$ \\
Synthesized beam & $8.0^{\prime \prime} \times 7.1^{\prime \prime}$ & $13.1^{\prime \prime} \times 10.8^{\prime \prime}$ \\
$\quad$ (PA) & $36^{\circ} .4$ & $35^{\circ} .2$ \\
Sensitivity $(\sigma)$ & $0.3 \mathrm{mJy}$ beam & $1.3 \mathrm{mJy} \mathrm{beam}{ }^{-1}$ \\
Dynamic range & $\sim 440$ & $\sim 410$ \\
Calibrator & $3 \mathrm{C} 147$ & $3 \mathrm{C} 147$ \\
$S_{v}$ (3C 147) & $38.26 \mathrm{Jy}$ & $59.14 \mathrm{Jy}$ \\
& & \\
\hline
\end{tabular}

similar in length to those of the VLA $D$-array, while the baselines between the arm antennas are comparable in length to the VLA $B$-array. A single observation with the GMRT hence yields information on both small and large angular scales. To enhance the low surface brightness features, the maps were therefore made at various scales. The morphology and spectra of 3C 129 and some of the other sources in the field are described below. In the discussion we have also made use of the VLA $330 \mathrm{MHz}$ image of the field kindly supplied by Lane and published in Lane et al. (2002). Since, maps published in Lane et al. (2002) are in B1950 epoch, we also present all our maps in the same epoch.

\subsection{Radio morphology of 3C 129}

This complex radio source shows a twin-tailed morphology at both frequencies. The maps illustrate the main characteristics of 3C 129 at low frequencies. The curving tail is at least $22^{\prime}$ long at $240 \mathrm{MHz}$ (Fig. 1), while at the higher frequency $\left(610 \mathrm{MHz}\right.$, Fig. 2) the tail is detectable out to $\sim 20^{\prime}$ from the head. This is because the spectrum steepens along the length of the tail and the extended outer components are not bright enough to be detected in high frequency observations (van Breugel 1982). The nuclear source of 3C 129 is unresolved at both frequencies. In both the radio maps, dissimilar behaviour of the two jets indicates that the upper jet is brighter than the lower. Using the radio morphology of them at 240 and $610 \mathrm{MHz}$, we further find that in the upper jet, the wiggles continue and lead to the far tail, while the lower jet ends earlier and disappears at a distance of $\sim 7^{\prime}$ from the nucleus. There is also a sharp drop in surface brightness at this position.

In Table 2, we show the integrated flux densities of 3C 129 and other extended sources in the field along with previous measurements at frequencies below $1500 \mathrm{MHz}$. Our estimate at $610 \mathrm{MHz}$ agrees well with that of Jägers (1987) got using the WSRT while our $240 \mathrm{MHz}$ is in reasonable agreement 
3C 129 at $240.188 \mathrm{MHz}$ GMRT resolution

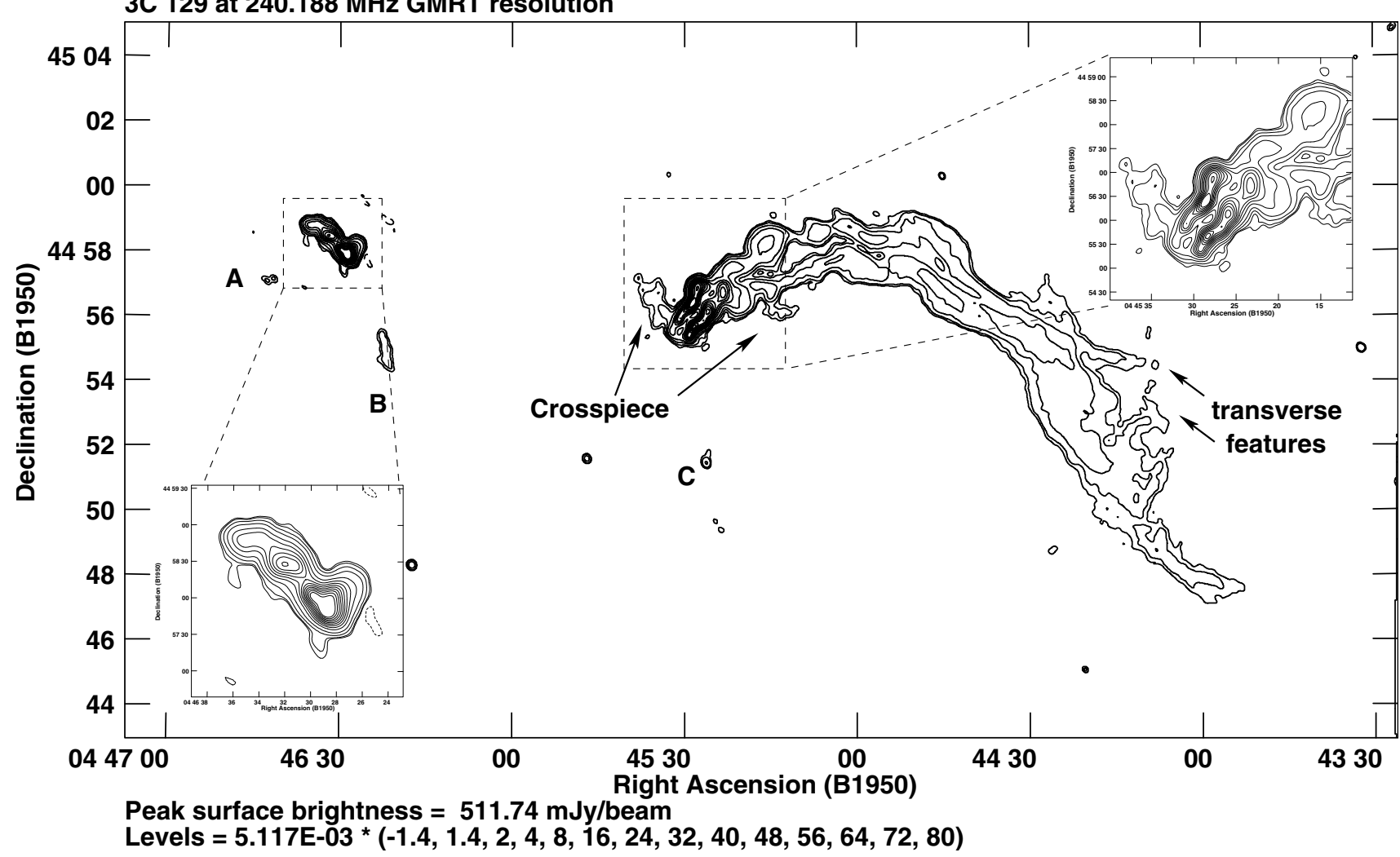

Fig. 1. Full synthesis GMRT map of 3C 129 and the sources in its field at $240 \mathrm{MHz}$. The two insets show 3C 129.1 (lower left) and head of 3C 129 (upper right). The CLEAN beam is $13^{\prime \prime} .1 \times 10^{\prime} .8$ at a PA of $35^{\circ} .2$.

with what is expected from its spectrum. We therefore believe that we have not lost any flux density in our interferometric observations.

\subsection{Radio morphology and integrated spectra of sources in the $3 C 129$ field}

The $20^{\prime}$ region around $3 \mathrm{C} 129$ has some interesting sources, like 3C 129.1, the crosspiece, etc., which we describe here.

3C 129.1: Our observations of 3C 129.1 show it to be an elongated radio source with angular size of $\sim 2^{\prime}$ along the north-east. High resolution observations at $2.7 \mathrm{GHz}$ by Downes (1980) suggest it to be a double source with a prominent core. The core and double structure are also seen in our $610 \mathrm{MHz}$ high resolution map. But at both 240 and $610 \mathrm{MHz}$, there is considerable diffuse emission around the source, some of which extends well beyond the double structures, typical of an Fanaroff-Riley type I (FR I) source (Fanaroff \& Riley 1974). The diffuse emission shows some curvature which is consistent with the suggestion by Lane et al. (2002) that it could be a wide-angle-tail source with the source moving with small transverse velocity, though it could also be due to buoyancy effects (see Sect. 6).

This radio source has a total flux density of $\sim 0.94 \mathrm{Jy}$ at $1400 \mathrm{MHz}$ (Condon et al. 1998) and our maps at 240 and $610 \mathrm{MHz}$ give a total flux density of 4.6 and $2.0 \mathrm{Jy}$ respectively. Its low frequency spectral index is, $\alpha_{240}^{610}=-0.86 \pm$ 0.25 which is typical of sources associated with clusters
(Baldwin \& Scott 1973). There is a weak gradient in spectral index behaviour along the source (Fig. 3); with centre which being flat is surrounded by steeper spectrum region.

The Crosspiece: Using WSRT, Jägers \& de Grijp (1983) at $608 \mathrm{MHz}$ reported a small projection near the head on one side of 3C 129. This projection has been confirmed by Lane et al. (2002) in their high resolution map at $330 \mathrm{MHz}$ who have named it as the "Crosspiece". We also detect this feature clearly at both the observed frequencies and see evidence for this feature in the NVSS at $1400 \mathrm{MHz}$. From its integrated flux densities, we estimate its spectral index $\alpha_{240}^{610}$ as $-1.04 \pm .22$ and is roughly straight between 240 and $1400 \mathrm{MHz}$. This value is flatter than the spectral index $\left(\alpha_{330}^{600}\right.$ of -3.0$)$ estimated by Lane et al. (2002), who had used the results of Jägers \& de Grijp (1983) at $608 \mathrm{MHz}$ and they seem to have underestimated its flux density.

Sources A, B and C: The faint sources A and B are seen in VLA maps at $74 \mathrm{MHz}$ and $330 \mathrm{MHz}$ (Lane et al. 2002). We detect sources $\mathrm{A}$ and $\mathrm{B}$ at $240 \mathrm{MHz}$, but not at $610 \mathrm{MHz}$. Source C has been seen in NVSS (Condon et al. 1998) and at $330 \mathrm{MHz}$ (Lane et al. 2002). We detect source C at both 240 and $610 \mathrm{MHz}$. It has an integrated flux density of 40.4 and $13.9 \mathrm{mJy}$ at 240 and $610 \mathrm{MHz}$ respectively. These detections of source C, give spectral indices $\alpha_{240}^{610}$ and $\alpha_{240}^{1400}$ as $-1.14 \pm 0.31$ and $-1.51 \pm 0.07$ respectively. Using detection at $240 \mathrm{MHz}$ and 
3C 129 at 616.562 MHz GMRT Resolution

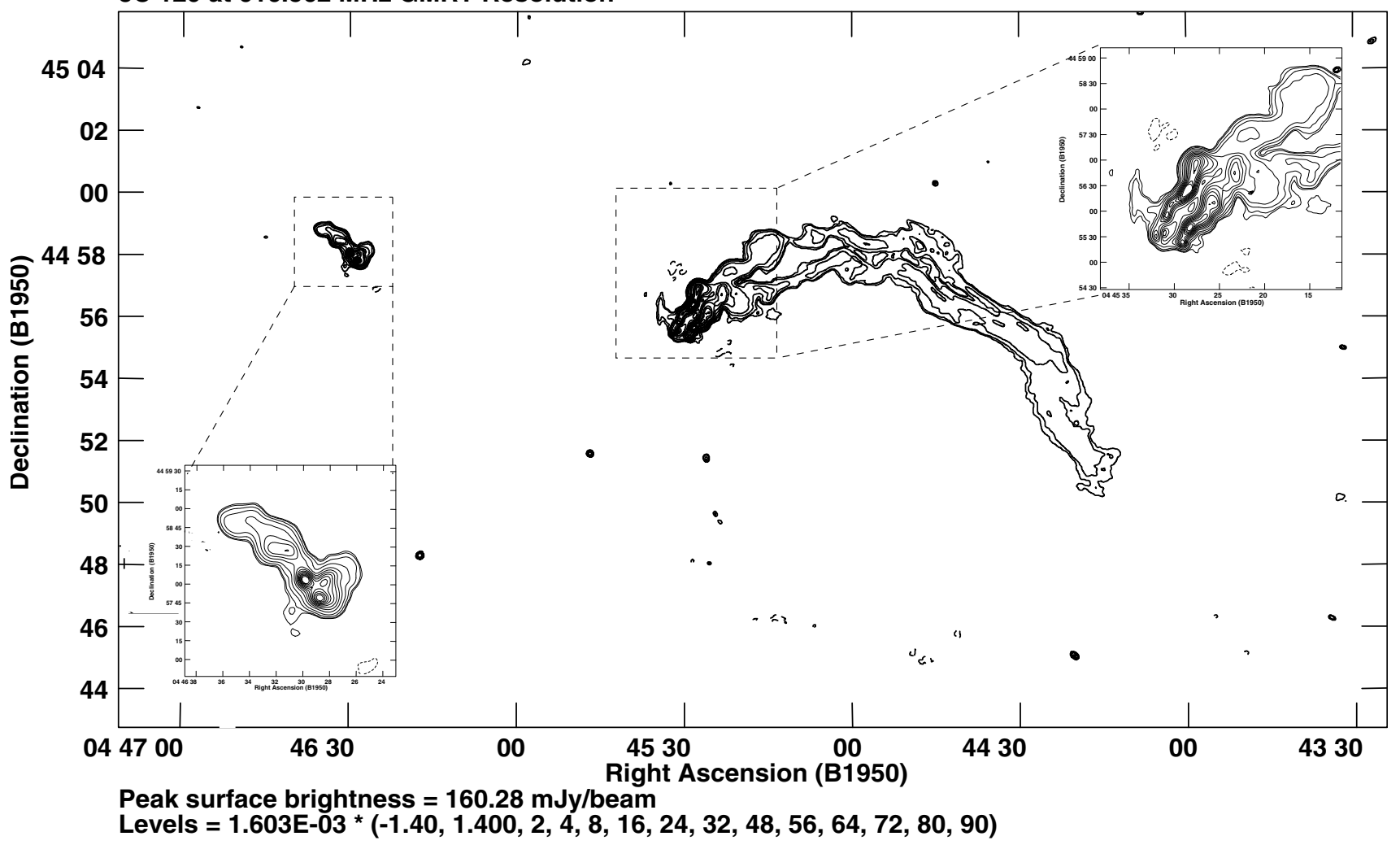

Fig. 2. Full synthesis GMRT map of 3C 129 and the sources in its field at $610 \mathrm{MHz}$. The two insets show 3C 129.1 (lower left) and head of 3C 129 (upper right). The CLEAN beam is $8^{\prime \prime} 0 \times 7$ 7' 1 at a P.A. of 36.4. The "cross" in the lower left inset marks the position of the optical nucleus with the size of the cross representing $1 \sigma$ uncertainty in position.

Table 2. The total intensity comparisons for 3C 129 and field sources. The flux densities quoted are in Jy along with corresponding errorbars $(1 \sigma)$. The $178 \mathrm{MHz}$ measurements are using large Cambridge interferometer (Ryle 1960), 325 and $1400 \mathrm{MHz}$ measurements are using VLA (Lane et al. 2002; Condon et al. 1998), and 240, $610 \mathrm{MHz}$ measurements are our observations; and II: Perley \& Erickson (1979), ${ }^{\S}$ : Bennett (1962) (the revised 3rd Cambridge catalogue, the flux integrated from map for 3C 129 and 3C 129.1 is $46.9 \pm 4.7$ Jy, Gower et al. 1967), ${ }^{\dagger}$ : Jägers (1987), and ${ }^{\ddagger}$ :White \& Becker (1992) (Green Bank, Northern Sky Survey). We also quote $3 \sigma$ values for the non-detections e.g. sources, A and $\mathrm{B}$ at $610 \mathrm{MHz}$, and Crosspiece, $\mathrm{A}, \mathrm{B}$ and $\mathrm{C}$ at $1400 \mathrm{MHz})$.

\begin{tabular}{|c|c|c|c|c|c|c|}
\hline & \multicolumn{6}{|c|}{ Flux density (Jy) } \\
\hline $3 \mathrm{C} 129$ & $90.0 \pm 5.0^{\mathbb{I I}}$ & $21.0^{\S}$ & $27.5 \pm 1.3$ & $21.2 \pm 1.4$ & $\begin{array}{r}14.5 \pm 1.4 \\
14.4 \pm 0.2^{\dagger}\end{array}$ & $\begin{array}{l}7.0 \pm 1.0 \\
5.3^{\ddagger}\end{array}$ \\
\hline 3C 129.1 & & $11.0^{\S}$ & $4.6 \pm 0.4$ & $3.5 \pm 0.8$ & $2.0 \pm 0.5$ & $0.9 \pm 0.4$ \\
\hline Crosspiece & & & $0.032 \pm 0.005$ & $0.020 \pm 0.003$ & $0.012 \pm 0.002$ & $<0.003$ \\
\hline $\mathrm{A}$ & & & $0.05 \pm 0.002$ & $0.03 \pm 0.002$ & $<0.003$ & $<0.003$ \\
\hline B & & & $0.14 \pm 0.005$ & $0.05 \pm 0.001$ & $<0.003$ & $<0.003$ \\
\hline $\mathrm{C}$ & & & $0.040 \pm 0.003$ & $0.03 \pm 0.004$ & $0.014 \pm 0.003$ & $<0.003$ \\
\hline
\end{tabular}

upper detection limits at $610 \mathrm{MHz}$ for the sources $\mathrm{A}$ and $\mathrm{B}$, we conclude that they are steeper than, $\alpha_{240}^{610},-3.0 \pm 0.3$ and $-4.1 \pm 0.3$ respectively.

\section{Low frequency spectra of $3 C 129$}

The observations and morphology described in the previous section (Sect. 3.1) allow us to investigate in detail the spectral index distribution of 3C 129. Both the GMRT $610 \mathrm{MHz}$ and VLA $330 \mathrm{MHz}$ maps were restored with the restoring beam corresponding to the $240 \mathrm{MHz}$ map (13'. $1 \times 10^{\prime} .8$ at PA 35.2). The final calibrated UV data at $610 \mathrm{MHz}$ was first mapped using UV taper 0-22 $\mathrm{k} \lambda$, which is similar to that of $240 \mathrm{MHz}$ data and then restored using the restoring beam corresponding to the $240 \mathrm{MHz}$ map. At $330 \mathrm{MHz}$, since we had only map, we restored it using restoring beam corresponding to that of 


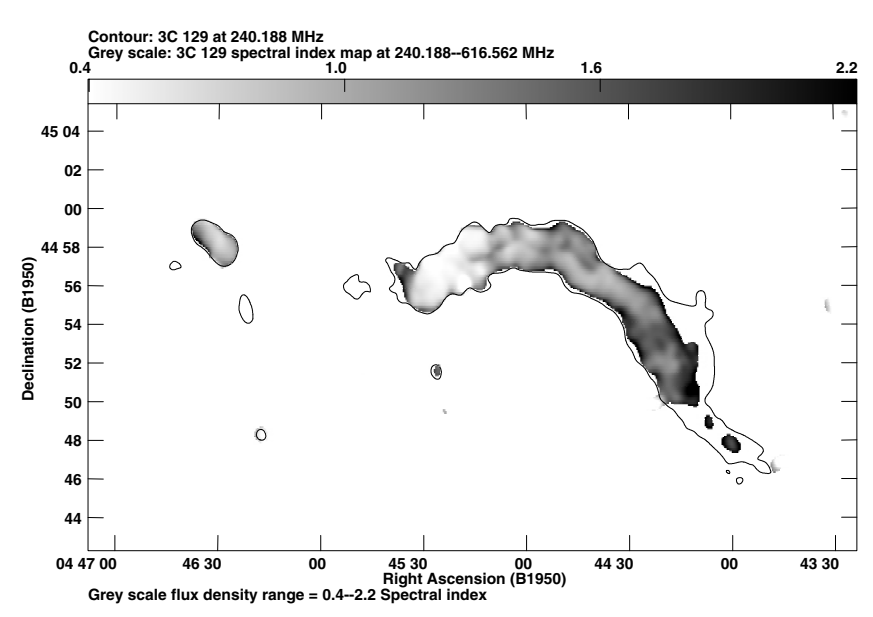

Fig. 3. Distribution of the spectral index $\alpha\left(S_{v} \propto v^{\alpha}\right)$ between 240, $330 \mathrm{MHz}$ (upper) and 240, $610 \mathrm{MHz}$ (lower) as computed from images made with a matched restoring beam $\left(36^{\prime \prime} 9 \times 28^{\prime \prime} .4\right.$ at PA $\left.=21^{\circ} .6\right)$. The lighter regions represent the relatively flat spectrum regions as compared to the darker regions which represent steep spectrum (although the full range of spectral index is -3.3 to 2.4 , we have shown only 0.4 to 2.2 for clarity). Also, plotted is the $3 \sigma$ contour corresponding to map at $240 \mathrm{MHz}$. The blank region inbetween closed contour and grey scale is probably steeper (or intermediate) than the shown darker regions in the grey scale plot.

$240 \mathrm{MHz}$ map. These three maps restored with the same resolution, GMRT 240 and $610 \mathrm{MHz}$ and VLA $330 \mathrm{MHz}$, were used for the spectral analysis. We determine the spectral index distribution using a number of techniques.

The standard, direct method of determining the spectral index between maps $S_{v_{1}}(x, y)$ and $S_{v_{2}}(x, y)$ at two frequencies $v_{1}$ and $v_{2}$ is given by

$\alpha_{v_{1}, v_{2}}(x, y) \equiv \frac{\log \left(S_{v_{1}} / S_{v_{2}}\right)}{\log \left(v_{1} / v_{2}\right)}$

We also looked at the spatially "filtered" images (Wright et al. 1999) and "spectral tomography" images (Rudnick 2002) to enhance weak features seen in the maps. We do not find evidence for a new feature emerging in the filtered or tomographic images, which could have been missed in the single frequency maps. While, it could also be due to sensitivity limits of our maps, this is possibly due to the fact that these techniques are effective in separating overlapping large-scale and small-scale features with different spectral distributions. Hence, these techniques are found to be useful for radio galaxies, supernova remnants, etc. (Rudnick 2002). Whereas, head-tail sources are understood to be FR I radio sources moving through the gas in the cluster and the shape of the source is due to the diffuse radio emitting plasma being decelerated by the intracluster medium (ICM) (Miley et al. 1972). Such sources do not suffer from regions with overlapping features with different spectral indices and therefore these techniques did not seem effective. However, in these series of slices through the source (in other words, spectral tomography images for a range of spectral indices), different parts of the source become prominent in different sets

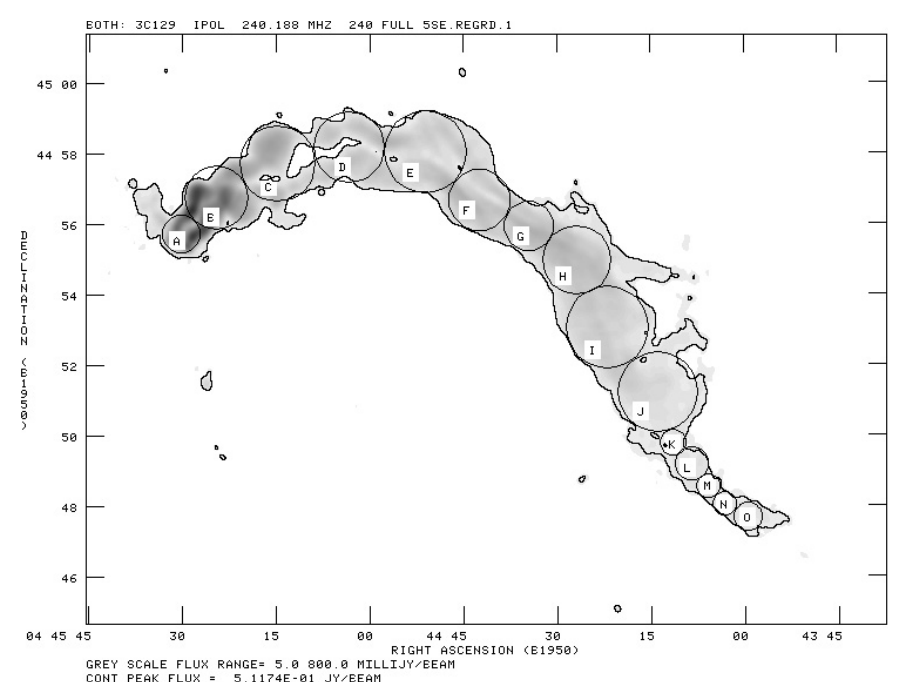

Fig. 4. Figure showing sampled 15 distinct regions along the ridge line of maximum emission. The size of each region correspond to the width of the source at the position of the region. These regions (marked, A, B, C, ... and O) are different from the field sources A, B and $\mathrm{C}$ discussed in previous section.

of images and provide additional support to their being real features.

In order to set more reliable estimates of the large scale behaviour of the source, we selected 15 circular regions (A, B, C, ... and $\mathrm{O}$ ), centered on the ridge lines of maximum emission of diameters corresponding to the width of the source at that position. The position and size of these regions are shown in Fig. 4. For each of these regions, we compute the total flux and construct the two frequency radio energy spectrum as a function of distance with respect to the head. Table 3 gives brightness (in units of $\mathrm{Jy} \mathrm{arcmin}^{-2}$ ) at 240, 330 and $610 \mathrm{MHz}$ for each of these regions. The distances in Col. 2 correspond to the distance of each of the regions, in arcmin, from the head along the tail. The profiles of the two frequency spectral indices, as a function of the distance from the head are shown in Fig. 5.

Figures 3 (spectral index distribution) and 5 (spectral index profile) shows that the spectra is flattest $(\alpha \gtrsim-0.5)$ at the core of 3C 129 in both, $\alpha_{240}^{610}$ and $\alpha_{240}^{330}$, spectral index maps. Also in the spectral index map at 240 and $610 \mathrm{MHz}$ (Fig. 3), the spectral index flattens slightly along the jet to a minimum which occurs around $2^{\prime}$ (see Fig. 5). Between $2^{\prime}$ and $7^{\prime}$ from the head, the spectrum of the source steepens from -0.5 to -0.8 ; from $7^{\prime}$ to $12^{\prime}$ the spectrum remains constant; and from $12^{\prime}$ up to the farthest tail end, spectral index steepens further from -0.8 to $\sim-2.0$. Whereas, in the low-frequency spectral index profile at 240 and $330 \mathrm{MHz}$, the region up to $2^{\prime}$ from core has a constant spectral index of -0.8 ; between $2^{\prime}$ and $7^{\prime}$ from the head, the spectrum of the source steepens from -0.8 to -1.2 ; from $7^{\prime}$ to $12^{\prime}$ the spectrum remains constant; and from $12^{\prime}$ to the farthest tail end, spectral index steepens further from -1.2 to $\sim 2.5$. Thus, the spectrum from our observations, roughly supports Pacholczyk \& Scott (1976) interpretation.

In terms of the electron spectrum between 240 and $610 \mathrm{MHz}$, the faint steepening of electron spectral index, $\gamma\left(N(E) \mathrm{d} E \propto E^{-\gamma} \mathrm{d} E\right.$ and $\left.\gamma=1-2 \alpha\right)$ is observed from the 
Table 3. Distance from the head along the tail (in arcmin), size (in arcmin), flux density of all the distinct regions at 240,330 and $610 \mathrm{MHz}$ (in $\mathrm{Jy}_{\mathrm{arcmin}}^{-2}$ ), and equipartition parameters at the sampled positions. The age (expressed in Myr) is calculated following formalism in Sect. 5.1 and using $v_{\mathrm{br}}$ of $610 \mathrm{MHz}$ (if $v_{\mathrm{br}}=1400 \mathrm{MHz}$, these would drop by a factor of $\sim 1.5$ ).

\begin{tabular}{|c|c|c|c|c|c|c|c|c|c|}
\hline & $\begin{array}{l}\text { Distance } \\
\left({ }^{\prime}\right)\end{array}$ & $\begin{array}{r}\text { Size } \\
\left({ }^{\prime}\right)\end{array}$ & $B_{240 \mathrm{MHz}}$ & $\begin{array}{c}B_{330 \mathrm{MHz}} \\
\left(\mathrm{Jy}^{\left.-\operatorname{arcmin}^{-2}\right)}\right)\end{array}$ & $B_{610 \mathrm{MHz}}$ & $\begin{array}{c}U_{\min } \\
\left(\mathrm{erg} \mathrm{cm}^{-3}\right)\end{array}$ & $\begin{array}{c}P_{\mathrm{eq}} \\
\left(\text { dyne } \mathrm{cm}^{-2}\right)\end{array}$ & $\begin{array}{c}B_{\mathrm{eq}} \\
(\mu \mathrm{G})\end{array}$ & $\begin{array}{r}\text { Age } \\
(\mathrm{Myr})\end{array}$ \\
\hline A & 0.00 & 1.07 & 4.444 & 3.492 & 2.458 & $5.6 \times 10^{-12}$ & $1.9 \times 10^{-12}$ & 7.7 & 58.7 \\
\hline B & 1.44 & 1.80 & 4.527 & 3.553 & 2.806 & $4.3 \times 10^{-12}$ & $1.4 \times 10^{-12}$ & 6.7 & 71.3 \\
\hline $\mathrm{C}$ & 3.42 & 2.13 & 1.089 & 0.778 & 0.622 & $1.7 \times 10^{-12}$ & $5.8 \times 10^{-13}$ & 4.2 & 125.3 \\
\hline $\mathrm{D}$ & 5.51 & 2.00 & 0.694 & 0.494 & 0.324 & $1.4 \times 10^{-12}$ & $4.6 \times 10^{-13}$ & 3.8 & 141.2 \\
\hline $\mathrm{E}$ & 7.68 & 2.33 & 0.728 & 0.474 & 0.278 & $1.3 \times 10^{-12}$ & $4.4 \times 10^{-13}$ & 3.7 & 145.7 \\
\hline $\mathrm{F}$ & 9.73 & 1.73 & 1.000 & 0.700 & 0.420 & $1.9 \times 10^{-12}$ & $6.2 \times 10^{-13}$ & 4.4 & 120.4 \\
\hline $\mathrm{G}$ & 11.31 & 1.40 & 1.174 & 0.807 & 0.510 & $2.3 \times 10^{-12}$ & $7.7 \times 10^{-13}$ & 4.9 & 106.2 \\
\hline $\mathrm{H}$ & 12.99 & 1.93 & 1.134 & 0.707 & 0.417 & $1.9 \times 10^{-12}$ & $6.3 \times 10^{-13}$ & 4.4 & 119.7 \\
\hline I & 15.08 & 2.33 & 0.744 & 0.428 & 0.236 & $1.3 \times 10^{-12}$ & $4.4 \times 10^{-13}$ & 3.7 & 144.8 \\
\hline $\mathrm{J}$ & 17.40 & 2.27 & 0.457 & 0.216 & 0.141 & $1.0 \times 10^{-12}$ & $3.4 \times 10^{-13}$ & 3.2 & 164.7 \\
\hline $\mathrm{K}$ & 18.90 & 0.73 & 0.325 & 0.146 & 0.012 & $1.6 \times 10^{-12}$ & $5.3 \times 10^{-13}$ & 4.1 & 131.0 \\
\hline $\mathrm{L}$ & 19.70 & 0.93 & 0.345 & 0.132 & 0.027 & $1.4 \times 10^{-12}$ & $4.8 \times 10^{-13}$ & 3.9 & 138.5 \\
\hline M & 20.49 & 0.67 & 0.360 & 0.141 & 0.027 & $1.8 \times 10^{-12}$ & $6.0 \times 10^{-13}$ & 4.3 & 123.1 \\
\hline $\mathrm{N}$ & 21.17 & 0.67 & 0.354 & 0.153 & 0.048 & $1.8 \times 10^{-12}$ & $5.9 \times 10^{-13}$ & 4.3 & 123.7 \\
\hline $\mathrm{O}$ & 21.94 & 0.80 & 0.337 & 0.135 & 0.075 & $1.6 \times 10^{-12}$ & $5.2 \times 10^{-13}$ & 4.0 & 133.1 \\
\hline
\end{tabular}
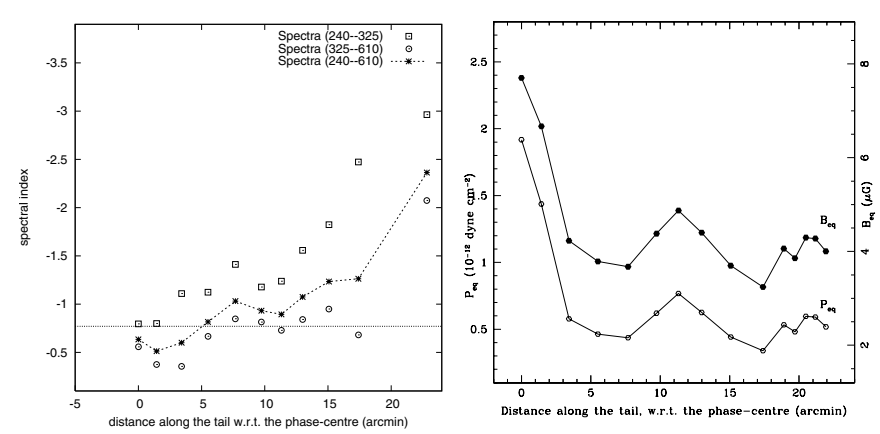

Fig. 5. Left: spectral index profile along the $3 \mathrm{C} 129$. The profile corresponds to the regions shown in Fig. 4. The horizontal line shows the averaged spectral index $(-0.77)$ using integrated flux density of the source. A single averaged value of spectral index positioned at the position of $\mathrm{O}$ is shown for all 5 regions, from $\mathrm{K}$ to $\mathrm{O}$, which are at the farther end of the tail. Errorbars $(1 \sigma)$ on the spectral indices are between 0.03 and 0.22 . Right: the minimum pressure $\left(\right.$ dyne $\left.\mathrm{cm}^{-2}\right)$ and equipartition magnetic field $(\mu \mathrm{G})$ of the radio plasma along the tail at the sampled positions.

head towards the tail. The electron spectrum steepens from 2 to 2.6 from region close to the core and up to $\sim 7^{\prime}$; from $7^{\prime}$ to $12^{\prime}$ the electron spectrum remains constant; and finally beyond $12^{\prime}$ results in even more steepening of the electron spectrum. This steepening of electron energy spectrum is due to synchrotron ageing of the radio plasma. The lowest energy electrons diffuse into the lower pressure regions and form the steep spectrum tail at the farther end from the head. This may be quite easy along the well ordered magnetic field of 3C 129 as suggested by van Breugel (1982).

Furthermore, the spectra of each of the distinct regions (Fig. 4) using the data (Table 3) is straight. Even on adding the upper detection limits at $1400 \mathrm{MHz}$ using published map by van Breugel (1982), the spectra of each of these components remain roughly straight. In other words, our data provides support to the notable result brought out by Perley \& Erickson (1979) that straight spectra are seen at all points down the tail.

\section{Physical parameters of the source}

Using the standard formulae (Pacholczyk 1970), we estimate the equipartition internal energy density $\left(U_{\min }\right)$, pressure $\left(P_{\mathrm{eq}}=\right.$ $\left.U_{\min } / 3\right)$, and magnetic energy $\left(B_{\text {eq }}\right)$ for the sampled regions in 3C 129 (see Table 3). We assume that the transverse size of each component in the source is the path length through the source along the line of sight.

Figure 5 shows the minimum pressure and equipartition magnetic field of 3C 129 plotted as a function of distance along the tail from the head. It therefore seems that the pressure in farther end of the tail is less by a factor of 4 as compared to the region close to core and hence is more diffuse.

\subsection{Source age}

The observed shape of the radio spectrum results from competition between energy injection and losses due to adiabatic expansion, synchrotron emission and inverse Compton (IC) scattering off CMB photons. Therefore, the shape of the radio spectrum along with a detailed understanding of these processes can yield the age of the source, the duration of initial particle injection and the magnetic field strength.

Following Miley (1980), we derive the upper limit to the age of the synchrotron electrons at several positions along the 
radio tail. The total radiative age is calculated as

$t=1060 \frac{B^{0.5}}{\left(B^{2}+B_{\mathrm{IC}}^{2}\right)}\left[(1+z) v_{\mathrm{br}}\right]^{-0.5} \mathrm{Myr}$,

where $v_{\mathrm{br}}$ is expressed in $\mathrm{GHz}$, and is the break frequency of the injected electron population, where the spectrum changes by 0.5 . The magnetic fields, $B$ and $B_{\mathrm{IC}}$, are expressed in $\mu \mathrm{G}$ and the radiative age, $t$, in Myr. We further choose $B=B_{\mathrm{IC}} / \sqrt{3}$, the value that gives maximum radiative lifetime (van der Laan \& Perola 1969) and $B_{\mathrm{IC}}=4(1+z)^{2} \mu \mathrm{G}$. With our sparse measurements for each of the regions, the break frequency, if it exists at all in 3C 129, must lie either above $610 \mathrm{MHz}$ or below $240 \mathrm{MHz}$. This is consistent with the larger extent suggested by Perley \& Erickson (1979), who suggested that the break frequency must be either above $1400 \mathrm{MHz}$ (and probably above $2695 \mathrm{MHz}$, Riley 1973) or below $43 \mathrm{MHz}$. We give age derived using equipartition magnetic field and using $610 \mathrm{MHz}$ as the break frequency at different locations along the source in Table 3. We find that age of each of the regions between " $\mathrm{C}$ " and "O" is within $~ 50$ Myrs. The core is the youngest with an age of $~ 59$ Myr. The assumption that there is equipartition of energy between the radiating particles and magnetic field seems to break at regions close to "J", where the age peaks as compared to the adjacent regions. Since, we have not determined $B_{\text {eq }}$ and $v_{\mathrm{br}}$ independently (Komissarov \& Gubanov 1994; Slee et al. 2001) and because the age of any region from 3 ' (region " $C$ ") onward is not very different from the age of the farthest region, labeled " $\mathrm{O}$ " in Table 3, we use the averaged age, i.e. $~ 124 \mathrm{Myr}$, of all the regions from " $\mathrm{C}$ " to "O" as the representative age of the 3C 129. Assuming $1400 \mathrm{MHz}$ (Perley \& Erickson 1979) as the break frequency, the age of 3C 129 is 86 Myr. Miley et al. (1972) suggests the age of 3C 129 to be of the order of $\sim 200 \mathrm{Myr}$, which is based on the length of the tail and galaxy velocity. Therefore, processes like particle acceleration or injection of new particles into the tail must be present. On the other hand, if the break frequency is $\sim 240 \mathrm{MHz}$, then the age estimate of 200 Myr for 3C 129 is in agreement with the age estimate of Miley et al. (1972). We understand that without knowing the history of the relativistic electron population, i.e. their current and past magnetic field environments, it is difficult to assign a well defined age to 3C 129 on the basis of spectral behaviour. Nevertheless, we assume that equipartition magnetic field holds good at most of regions along the jet. The age of $200 \mathrm{Myr}$ therefore seems plausible for 3C 129 as suggested by Miley et al. (1972) and break frequency occurs at $\sim 240 \mathrm{MHz}$.

\section{Discussion}

The low frequency observations combined with recent observations at other wavelengths raise a number of questions regarding the nature and origins of the various sources in the field. Some of the earlier scenarios that were based on limited information need to be revised. In this section we will discuss the implications of the new observations on our understanding of the source.
Nature of the Crosspiece: The integrated radio flux densities of relic sources fall very rapidly with frequency, with power-law slopes, $\alpha$, between -2.1 and -4.4 near $1400 \mathrm{MHz}$ (Slee et al. 1994). Their break frequency is below $100 \mathrm{MHz}$ with typical age of $\sim 300 \mathrm{Myr}$ (e.g. archetype radio relic, Abell 85; Slee et al. 2001). Although the crosspiece has low surface brightness, we find that the spectral index of it is not very different from usual radio plasma outflow of 3C 129 (Sect. 3.2). We also find that the spectral index, between 240 and $1400 \mathrm{MHz}$, of it is roughly straight. The scenario proposed by Lane et al. (2002) in which the crosspiece is a steep spectrum relic source that has been reactivated by 3C 129 plowing and compressing it seems to be untenable since the adiabatic compression process cannot change the spectrum of the source. Close examination of the tail of 3C 129 reveals features extending perpendicular to the tail, similar to crosspiece, further out along the tail (see transverse features close to regions "H" and "J", shown in Fig. 1). Such features seem to be an integral part of the tail of 3C 129 and could arise due to perturbations in the jet and/or may be due to buoyancy effects because of density inhomogeneities in ICM. Furthermore, since the spectral index along the tail and at the crosspiece are similar and if we assume, following Lane et al. (2002), that the break frequency is at $1400 \mathrm{MHz}$, the maximum radiative age of the crosspiece is $\sim 60 \mathrm{Myr}$. This does not seems to agree with suggested dynamical age of about 40 Myr (Jägers \& de Grijp 1983). Also, an age of $40 \mathrm{Myr}$ along with an infall velocity of $1630 \mathrm{~km} \mathrm{~s}^{-1}$ (Lane et al. 2002) for 3C 129, would put crosspiece at a distance of at least 8!3 from the head. Hence, crosspiece being one-sided ejection of plasmoids or beams, which took place about 40 Myr (Jägers \& de Grijp 1983) is also unlikely. We therefore believe that the features like crosspiece are a manifestation of inhomogeneities in the medium and/or perturbations in the jet.

Kinematics of 3C 129: The galaxies associated with 3C 129 and 3C 129.1 (Hill \& Longair 1971) are approximately 0.7 and $1.05 \mathrm{mag}$ ( $B$-band) fainter than the brightest cluster member (Sandage 1975) which is situated about $\sim 5^{\prime}$ south of 3C 129. Recently Nilsson et al. (2000, see also Byrd \& Valtonen 1978) identified the brightest member to be the cluster centre and they developed a detailed kinematic model to explain the morphology of 3C 129, after account has been taken of the buoyancy. In the canonical picture, a head-tail source is FR I radio source in a cluster of galaxies and the characteristic shape is explained by the kinematics of the source and the properties of the beams (Fabian et al. 2002). Usually, the motion of the source is believed to be around the cluster center, which is the dominant gravitational force in the cluster. The centre of the cluster potential is also the location of X-ray emission due to the trapping of the hot ICM and is also the location of the brightest and most massive $\mathrm{cD}$ galaxy which is often a powerful radio galaxy (Riley 1975; Bahcall 1977; Fabian 1994). The observations of 3C 129 and its surroundings suggest that for this system, the picture is more complicated than the canonical picture; in particular, the kinematical centre and 
the centre of the X-ray emission for this cluster are different, which we discuss below:

Cluster centre: The X-ray emission is elliptical $\left(\sim 10^{\prime} \times \sim 6^{\prime}\right.$ with major in the east-west direction) and it peaks at and around the location of two discrete sources: an extended source near the core of the $3 \mathrm{C} 129.1$ and a point source at $2^{\prime}$ towards the south-west of the 3C 129.1. Although this point source seems to be at the centroid of X-ray emission, the location of the cluster centre is assumed to be between these two sources (Krawczynski et al. 2003). If 3C 129.1 is at the centre of the cluster, one needs to explain why it is not a $\mathrm{cD}$ galaxy and the dominant member of the cluster? It is possible that the mass of its optical host could be underestimated because of heavy obscuration (Downes 1980), hence the centre of the cluster could not be determined from optical observations. It is also possible that cluster is still in the process of growing via cannibalism and becoming a cD galaxy (McGlynn \& Ostriker 1980; Dressler 1984). However, identification of 3C 129.1 with the cluster centre poses problems for the kinematic model which explains the morphology of 3C 129. Since, if 3C 129 is going around the cluster centre or falling into it, it is not possible to generate the observed shape from kinematic models.

On the other hand, we probably do not understand the radio morphology of 3C 129.1 and faint sources A, B and C may also be playing an important role. The faint sources A and B are not associated with any known optical sources. We find that the source A has very steep spectrum $\left(\alpha_{240}^{610} \lesssim-3.0 \pm 0.3\right)$ and could be a radio relic source. Whereas, the source $\mathrm{C}$ is coincident with the optical galaxy WEIN 047 and which is also an IRAS source. This galaxy is detected in all the bands $(J$, $H, K_{\mathrm{s}}$ ) of 2 Metre All Sky Survey and is probably a starburst galaxy. The radio spectral index, $\alpha_{240}^{1400}=-1.51 \pm 0.11$ is typical of star-forming galaxies (e.g. M 82: Kellermann et al. 1971). Careful look at the features in the maps of 3C 129.1 at 240 and $610 \mathrm{MHz}$ reveal that its morphology is similar to most FR I radio galaxies, with bright regions on either side of the optical core (see our Fig. 2, Fig. $5(8.6 \mathrm{GHz})$ and Fig. $6(4.9 \mathrm{GHz})$ in Taylor et al. 2001, and Fig. 2 (2.7 GHz) in Downes 1980) that fade with distance from the core. Considering the fact that the source B is $\sim 3^{\prime}$ south of the optical core and the farthermost north-eastern feature of $3 \mathrm{C} 129.1$ is $\gtrsim 1^{\prime}$ from it, and their spectral indices, $\alpha_{240}^{610}$, are $\lesssim-4.1 \pm 0.3$ and $-1.8 \pm 0.6$ respectively, suggest that the spectrum steepens along the jets and the steepening of spectrum is due to synchrotron ageing of radio plasma. Furthermore, the bright regions seen on either side of the optical core and a sharp boundary on its south west side are either because of episodic activity in the core or because of buoyancy effects due to density inhomogeneities in the ICM. Therefore, it seems that the source $\mathrm{B}$ is probably a part of southern jet of 3C 129.1. This picture then suggests that 3C 129.1 is a wideangle-tail source as suggested earlier by Lane et al. (2002).

\section{Summary}

We have presented the lowest frequency image of 3C 129 at $240 \mathrm{MHz}$. The important consequences of these observations combined with our $610 \mathrm{MHz}$ observations and VLA map of Lane et al. (2002) at $330 \mathrm{MHz}$ are as follows:

1. The morphology of 3C 129 is similar to that of twin-tailed head-tail sources. Our both maps show curving tail detected out to $22^{\prime}$ from the head at $240 \mathrm{MHz}$ and up to $\sim 20^{\prime}$ at $610 \mathrm{MHz}$.

2. 3C 129.1 by itself, is unlikely to be a twin-tailed source and it being a head-tail source is also doubtful. Barring projection effects, it could be similar to that of bent-double sources. But if source B is probably a part of southern jet of $3 \mathrm{C} 129.1$, then it could be a FR I radio galaxy which is a wide-angle-tail source as suggested earlier by Lane et al. (2002).

3. The suggested scenario by Lane et al. (2002) for the crosspiece is unlikely. Its relatively flat spectrum ( $\alpha=$ $-1.04 \pm 0.22$ ) is not consistent with a fossil radio plasma revived by 3C 129's bow shock. We also do not believe Jägers \& de Grijp (1983) that crosspiece is one-sided ejection of plasmoids or beams. Instead it seems to have formed due to irregularities and inhomogeneities in the medium and/or perturbations in the jet.

4. The low frequency spectra between 240 and $610 \mathrm{MHz}$, interpreted as a synchrotron ageing, consist of four regimes along the tail: (a) region between $0^{\prime}$ and $2^{\prime}$ where the spectrum flattens from the head; (b) region between $2^{\prime}$ and $7^{\prime}$ where the spectrum steepens from -0.5 to -0.8 ; (c) a region between $7^{\prime}$ and $12^{\prime}$ where spectral index remains nearly constant; and (d) a region beyond 12' where further steepening occurs. Such a behaviour was earlier noted by Pacholczyk \& Scott (1976). We also confirm the result of Perley \& Erickson (1979) that straight spectra are seen at all points down the tail.

5. Assuming equipartition magnetic field and the break frequency occurs at $\sim 240 \mathrm{MHz}$, our age estimate of $\sim 200 \mathrm{Myr}$ seems plausible for 3C 129. This is in agreement to the age estimated by Miley et al. (1972).

6. There is some ambiguity as to the location of the cluster centre. While it is more probable that it is near 3C 129.1, near the centroid of the X-ray emission, locating it near the brightest galaxy in the cluster has the advantage of explaining the kinematics of 3C 129. Deep optical and infrared images would be useful in independently establishing the cluster centre.

Acknowledgements. We thank the staff of the GMRT who have made these observations possible. GMRT is run by the National Centre for Radio Astrophysics of the Tata Institute of Fundamental Research. We thank Wendy Lane and her collaborators for the $330 \mathrm{MHz}$ VLA map. D. V. L. thanks R. Nityananda, S. Rawlings, S. Roy and D. J. Saikia for discussions and several useful comments. This research has made use of the NASA/IPAC Extragalactic Database which is operated by the Jet Propulsion Laboratory, Caltech, under contract with the NASA.

\section{References}

Ananthakrishnan, S., \& Rao, A. P. 2002, Proc. of the Conf., Multi Colour Universe, held at the TIFR, India, 233

Baldwin, J. E., \& Scott, P. F. 1973, MNRAS, 165, 259 
Baars, J. W. M., Genzel, R., Pauliny-Toth, I. I. K., \& Witzel, A. 1977, A\&A, 61, 99

Bahcall, N. A. 1977, ARA\&A, 15, 505

Bennett, A. S. 1962, Mem. R. Astron. Soc, 68, 163

Bologna J. M., McClain, E. F., \& Sloanaker, R. M. 1969, ApJ, 156, 815

van Breugel, M. 1982, A\&A, 110, 225

van Breugel, M, \& Willis, A. G. 1981, A\&A, 96, 332

Byrd, G., \& Valtonen, M. J. 1978, ApJ, 221, 481

Condon, J. J., Cotton, W. D., Greisen, E. W., et al. 1998, AJ, 115, 1693

Downes, A. 1980, MNRAS, 190, 261

Downes, A. 1984, MNRAS, 211, 215

Dressler, A. 1984, ARA\&A, 22, 185

Fabian, A. C. 1994, ARA\&A, 32, 277

Fabian, A. C., Celotti, A., Blundell, K. M., Kassim, N. E., \& Perley, R. A. 2002, MNRAS, 331, 369

Fanaroff, B. L., \& Riley, J. M. 1974, MNRAS, 167P, 31

Gower, J. F. R., Scott, P. F., \& Wills, D. 1967, Mem. R. Astron. Soc, 71,49

Hill, J. M., \& Longair, M. S. 1971, MNRAS, 154, 125

Jaffe, W. J., \& Perola, G. C. 1973, A\&A, 26, 423

Jägers, W. J. 1987, A\&AS, 71, 603

Jägers, A. J., \& de Grijp, M. H. K. 1983, A\&A, 127, 235

Kardashev, N. S. 1962, Soviet Astron. 6, 317

Kellermann, K. I., \& Pauliny-Toth, I. I. K. 1971, Astrophys. Lett., 8, 153

Komissarov, S. S., \& Gubanov, A. G. 1994, A\&A, 285, 27

Krawczynski, H., Harris, D. E., Grossman, R., et al. 2003, MNRAS, 345,1255

van der Laan, H., \& Perola, G. C. 1969, A\&A, 3, 468

Lane, W. M., Kassim, N. E., Ensslin, T. A., Harris, D. E., \& Perley, R. A. 2002, AJ, 123, 2985
Macdonald, G. H., Neville, A. C., \& Ryle, M. 1966, Nature, 211, 1241 McGlynn, T. A., \& Ostriker, J. P. 1980, ApJ, 241, 915

Miley, G. K., Perola, G. C., van der Kruit, P. C., \& van der Laan, H. 1972, Nature, 237, 269

Miley, G. K. 1980, ARA\&A, 18, 165

Nilsson, K., Valtonen, M., Zheng, J.-Q., et al. 2000, IAU Coll. 174, ed. Valtonen, M. J., \& C. Flynn (San Francsico: ASP), ASP Conf. Ser., 209, 408

Owen, F. N., Burns, J. O., Rudnick, L., \& Greisen, E. W. 1979, ApJ, 229, L5

Pacholczyk, A. G. 1970, Radio Astrophysics (San Francisco: W. E. Freeman \& Co.)

Pacholczyk, A. G., \& Scott, J. S. 1976, ApJ, 203, 313

Perley, R. A., \& Erickson, W. C. 1979, ApJS, 41, 131

Riley, J. M. 1973, MNRAS, 161, 167

Riley, J. M. 1975, MNRAS, 170, 53

Rudnick, L., \& Burns, J. O. 1981, ApJ, 246, L69

Rudnick, L. 2002, PASP, 114, 427

Ryle, M. 1960, J. Instn elect. Engrs, 6, 14

Sandage, A. R. 1975, PASP, 87, 853

Slee, O. B., Roy, A. L., Murgia, M., Andernach, H., \& Ehle, M. 2001, AJ, 122, 1172

Slee, O. B., Roy, A. L., \& Savage, A. 1994, AuJPh, 47, 145

Swarup, G., Ananthakrishnan, S., Kapahi, V. K., et al. 1991, Cu. Sc., 60, 95

Taylor, G. B., Govoni, F., Allen, S. A., \& Fabian, A. C. 2001, MNRAS, 326,2

White, R. L., \& Becker, R. H. 1992, ApJS, 79, 331

Wright, M., Dickel, J., Koralesky, B., \& Rudnick, L. 1999, ApJ, 518, 284 